\title{
Technical Developments at the KIT Gyrotron Test Facility
}

\author{
Martin Schmid $^{\mathrm{a}}$, Volker Erckmann ${ }^{\mathrm{c}}$, Gerd Gantenbein ${ }^{\mathrm{a}}$, Stefan Illy ${ }^{\mathrm{a}}$, Stefan Kern ${ }^{\mathrm{a}}$, Christophe Liévin ${ }^{\mathrm{d}}$, \\ Andrey Samartsev ${ }^{\mathrm{a}}$, Andreas Schlaich ${ }^{\mathrm{b}}$, Thomáz Rzesnicki ${ }^{\mathrm{a}}$, Manfred Thumm ${ }^{\mathrm{a}, \mathrm{b}}$ \\ Karlsruhe Institute of Technology, Association EURATOM-KIT, Karlsruhe, D \\ ${ }^{a}$ Institute for Pulsed Power and Microwave Technology (IHM) \\ ${ }^{b}$ Institute of High Frequency Techniques and Electronics (IHE) \\ ${ }^{c}$ Max-Planck-Institute for Plasmaphysics, Association EURATOM-IPP, Greifswald, D \\ ${ }^{d}$ Thales Electron Devices, Vélicy, $F$
}

\begin{abstract}
Parasitic beam tunnel oscillations have been discovered on all gyrotrons currently being developed and tested at KIT (1MW/140 GHz tube for W7-X, $2 \mathrm{MW} / 170 \mathrm{GHz}$ coaxial gyrotron and 105-143 GHz frequency step tunable gyrotron). In the case of the W7-X gyrotrons these problems have been observed only on some of the series production tubes. A modified beam tunnel design, technologically close to the old design, has successfully been tested on both the coaxial and f-step-tunable gyrotrons and has subsequently been implemented on one of the W7-X series-production-tubes presently undergoing factory acceptance tests in Karlsruhe.

The ECRH test loads at KIT are operated under normal atmospheric conditions. Several loads have eventually failed in $1 \mathrm{MW}$ long pulse experiments and KIT has therefore started to design its own loads. The first KIT-load is based on a fixed conical mirror and a cylindrical aluminum absorber coated with a lossy material. The new load was very economical to manufacture and has so far successfully been used during the acceptance tests of two 1MW CW gyrotrons. Nevertheless a new load based on (uncoated) stainless steel absorbers is being developed as a backup solution for the ongoing high priority gyrotron testing.

A superconducting magnet capable of rapid field changes between 4.15 and 5.67 Tesla for frequency steptunable gyrotrons has been procured, has achieved its specified maximum (static) field of 7.2 Tesla and has also demonstrated its capability of rapid field-changes. Final acceptance however has been delayed pending rectification of problems with the magnetic field alignment.
\end{abstract}

Keywords: ECRH; gyrotron; beam tunnel; test load; superconducting magnet

\section{Parasitic Oscillations in W7-X gyrotrons}

During the ongoing series production of the W7-X gyrotrons problems with parasitic oscillations have been encountered, which prevented operation at nominal power. This led to acceptance test failures of a number (but not all) of the tubes [1]. Similar problems with beam tunnel oscillations have been identified on both the frequency step tunable gyrotron [2] and the European coaxial pre-prototype $170 \mathrm{GHz}$ gyrotron [3]. It is fair to say that quite an odyssey had to be gone through before the nature of the problem was recognized. It emerged eventually that parasitic oscillations in the electron-beam tunnel prevented operation at full power. Only with the development of a more sophisticated frequency measurement diagnostic system [4] a fair degree of confidence in the localization of the parasitic oscillations has become possible, together with an understanding of the nature of these parasitic oscillations.

The original Beam Tunnel consisted of a sandwiched structure of ceramic $(\mathrm{BeO})$-rings interleaved with copper rings (Fig.1). The first 3 gyrotrons worked well with output power in excess of $900 \mathrm{kWs}$. A forced ceramicssupplier-change seems to have been sufficient to alter the damping properties of this beam tunnel so much that that under otherwise identical operating conditions unwanted parasitic oscillations would be exited, particularly when approaching the designed gyrotron output power.
Several solutions were considered to suppress the unwanted parasitic oscillations, which were identified as backward waves being exited in the beam tunnel below the cavity. In follow-up inspections of rejected gyrotrons damage to the ceramics has been observed in the shaded region indicated in Fig.1

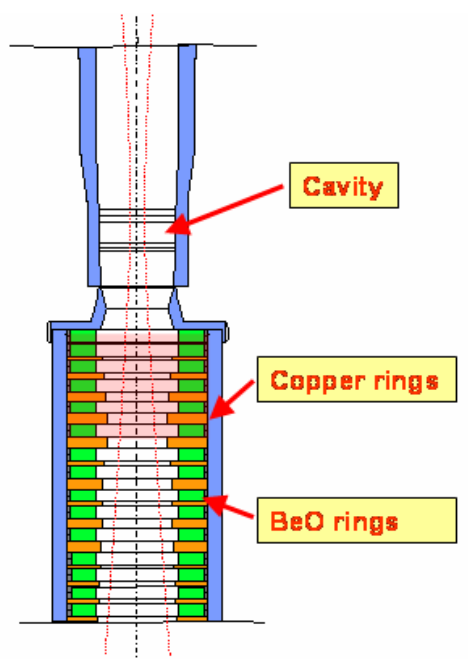

Fig.1 Original Beam Tunnel of the W7-X gyrotron

The considered solutions were:

a) implementation of a lossy ceramic beam tunnel (a solution employed for example in Japanese gyrotrons) 
b) breaking the azimuthal symmetry by introducing corrugated copper rings, avoiding symmetric $\mathrm{TE}_{0, \mathrm{p}}$-modes

c) using a monotonously conical shaped beamtunnel, thus avoiding cavity-like structures

All 3 solutions were prepared and a new beam tunnel combining solution b) and c) was designed (see Fig. 2) and tested in a one-to-one comparison on the frequency step tunable gyrotron and also on the coaxial preprototype gyrotron. This choice was involving least technological changes and avoided difficult quality control issues on the loss-properties of a ceramic beam tunnel. The solution worked very well and parasitic oscillations in the beam tunnel ceased completely in both gyrotrons.

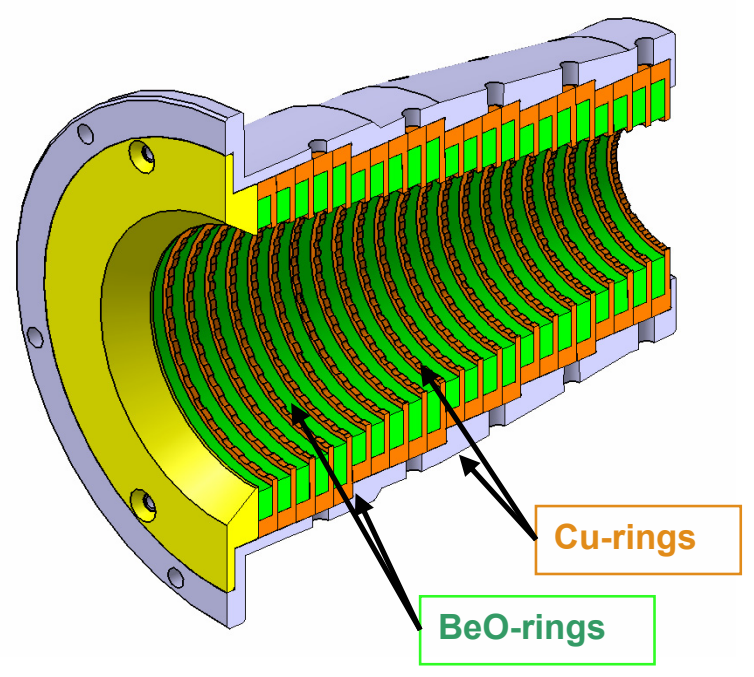

Fig.2 Improved Beam tunnel with corrugated copper rings and a conical contour

However, this may not yet be the end of all parasitic oscillations. While on the coaxial pre-prototype gyrotron no more parasitic modes could be seen and a record power of $2.2 \mathrm{MW}$ with $96 \%$ Gaussian content has been achieved [3], there was one remaining parasitic mode observed on the f-step-tunable tube, at around $135 \mathrm{GHz}$. Careful analysis showed that this parasitic mode is not emanating from the beam tunnel, but is actually a forward wave in the launcher, having the same indices (namely $\mathrm{TE}_{22,8}$ ) as the proper (wanted) cavity mode. It is thought that this parasitic mode may feed itself from the spent electron beam, without its own re-bunching process. First experiments on the W7-X series tube 004R (the first one equipped with the new beam tunnel) have also shown the presence of a parasitic oscillation at around $130.5 \mathrm{GHz}$ when operating at a power level above $800 \mathrm{~kW}$. While it is already clear that this mode is not exited in the beam tunnel, it has not yet been possible to define its precise origin. It could be either an After Cavity Interaction (ACI, see [5]) in the launcher or also a backward-wave interaction in the area of the connection between beam-tunnel and resonator (including down taper). The answer depends very critically on the precise magnetic field distribution in this area and detailed analysis is still ongoing.

\section{ECRH Test loads developed at KIT}

The KIT ECRH testbed is using a quasi optical transmission line in order to transfer the power from the gyrotron to the load, where the power can be measured calorimetrically (see fig. 3). The load is operated under normal atmospheric conditions and with a circularly polarized beam, which is important for homogenous absorption inside the lossy surface coating of the load. Operation under vacuum would be an advantage, but would necessitate an additional vacuum window or the use of closed waveguide between gyrotron and load. The former is expensive and the latter is likely to cause problems with the gyrotron operation, since all the reflected power from the load (some $3 \%$ on a good load) would be reflected back into the gyrotron, which can be extremely harmful to its proper functioning.

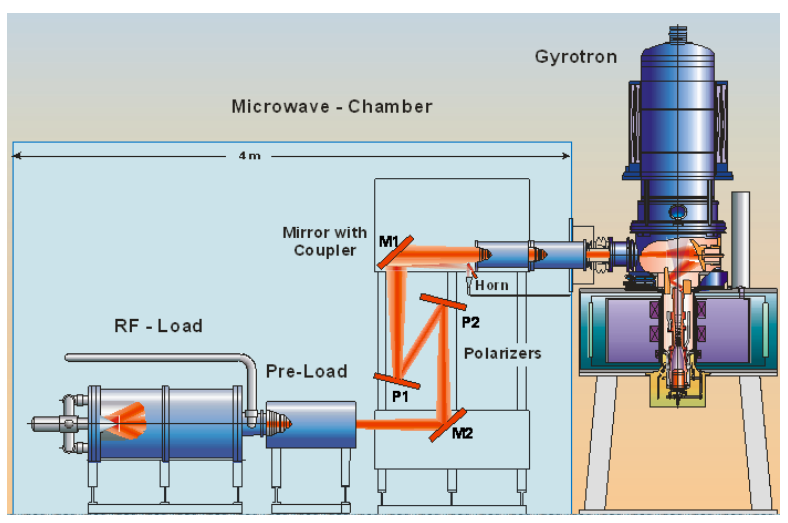

Fig.3 Transmission of gyrotron-beam to testload

Fig.4 is showing the basic principle of the load. The ECRH beam enters from the right through a narrow entrance (near the beam waste). It is then defocused by a fixed conical mirror on the opposite side and is gradually being absorbed in multiple reflections on the lossy surface of the inner cylinder wall of the load.

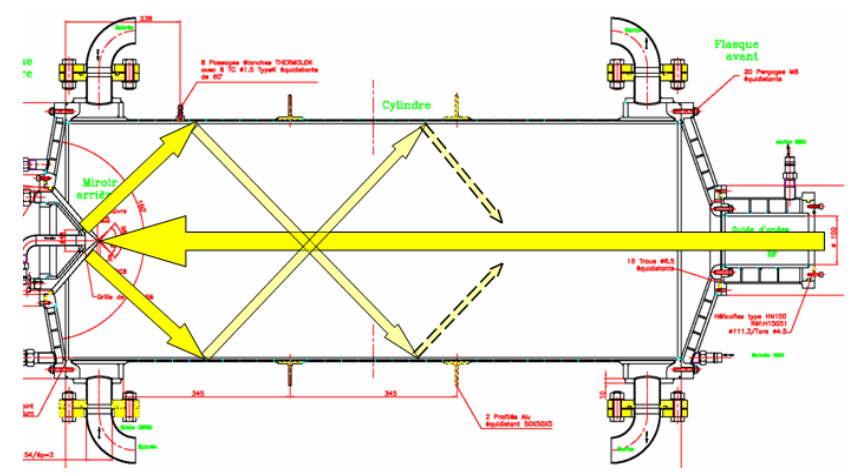

Fig.4 Early design of load with $\mathrm{TiO}-\mathrm{Al}_{2} \mathrm{O}_{3}$-coating (on a layer of $\mathrm{NiCrAlY}$ ) and a conical defocusing mirror (left)

The coating thickness is carefully controlled along the wall surface in order to obtain constant wall loading along the axis of the load. Fig.5 is showing a plot of the 
ray-tracing simulation of the power density on the inner cylinder of the load.

All parts of the load are double walled and a water flow rate of approx. $550 \mathrm{l} / \mathrm{min}$ is required to remove the dissipated power of $1 \mathrm{MW}$ in long-pulse and $\mathrm{CW}$ operation. The early design of the load shown in Fig.4 actually failed after a while with water leaks caused by thermal fatigue-cracks, because the double-wall structure used for the outer cylinder could not handle the differential expansion which occurred between the inner cylinder (heated by the RF) and the outer cylinder (which remains cool due to the heavy water flow). Based on the knowledge that the microwave design of the load is sound, a new load has been designed, using the same RF-design, but with a new concept for a separate inner

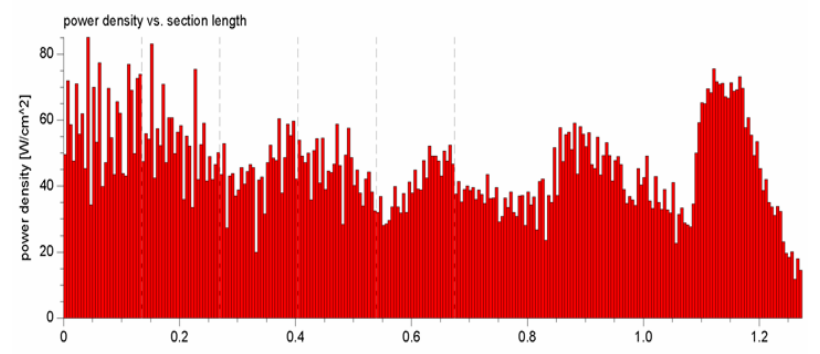

Fig.5 Power density along outer cylinder wall (simulated). The peak on the right is the first bounce of the beam reflecting off the conical mirror.

and outer cylinder, which can expand differentially against each other without causing undue stresses. Fig.6 shows a cross-section through the new load. The inner cylinder (aluminium-alloy) is castellated on the water side and has a wall-thickness of 12 / $16 \mathrm{~mm}$, so required to sustain the forces resulting from the water cooling circuit pressure of up to 6 bar. The differential movement between inner and outer cylinder against each other is taken up by Schnorr disks (visible on the right side of fig.6), which press the input plate against the inner cylinder with a near constant pressure. A sliding Oring preserves the tightness of the cooling circuit. This new load, designed and built very economically at KIT, is now successfully in use on the acceptance tests of its second $1 \mathrm{MW}$ gyrotron.

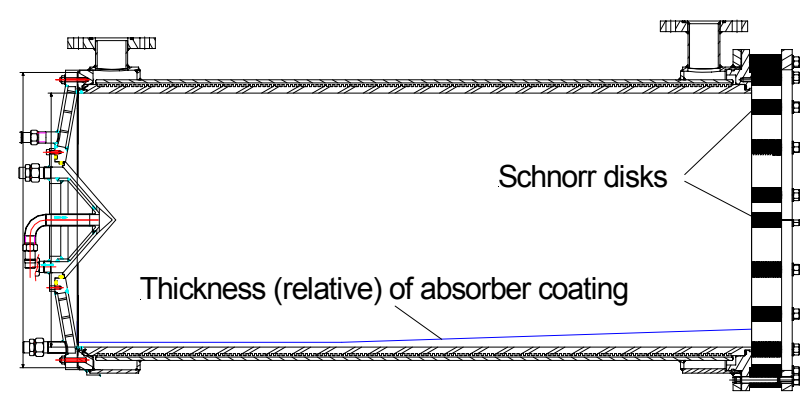

Fig.6 KIT version of $1 \mathrm{MW}$ load with $\mathrm{TiO}-\mathrm{Al}_{2} \mathrm{O}_{3}$-coating

A faultlessly working load is essential for long-pulsetesting of gyrotrons. The loss-coating is clearly one weakness in these loads, as for example misalignment could lead to excessive power densities and therefore coating-damage. Also, arcs which can not be avoided all the time, have the potential to damage the coating. Previous experience with the coating employed on these loads has shown that such damage can not be repaired easily (other types of coatings on other loads could just locally be removed and the load could be used again, all be it with lower absorption, but with $\mathrm{TiO}-\mathrm{Al}_{2} \mathrm{O}_{3}$-coating on a NiCrAlY layer this technique did not work, there was persistent arcing along the edge where the coating had been removed).

A load without coating may be more robust and easier to repair if damaged. In order to have a backup-load and also encouraged by good reports of such loads used elsewhere, KIT has started the development of an all stainless steel load. A first such load with a novel internal structure but without active cooling has been tested and has shown unexpected low levels of reflection $(<2 \%)$ and could be used with pulses up to 2 seconds at power levels of up to $700 \mathrm{~kW}$. Wall temperatures in excess of $250^{\circ} \mathrm{C}$ were reached (in successive pulses) before arcing developed. Arc damage in the form of very small pitting was minimal, but nevertheless had to be polished off in order to regain maximum power levels. Access to the internal structure therefore appears to be essential. Work is underway now to design a fully watercooled version of this load. In a first step, only the inner structure has been equipped with cooling. Such a load (Fig.6) is ready for testing with a $1 \mathrm{MW}$ CW gyrotron. If tests are successful, a completely cooled load will be manufactured as a back-up for the KIT gyrotron testbed.

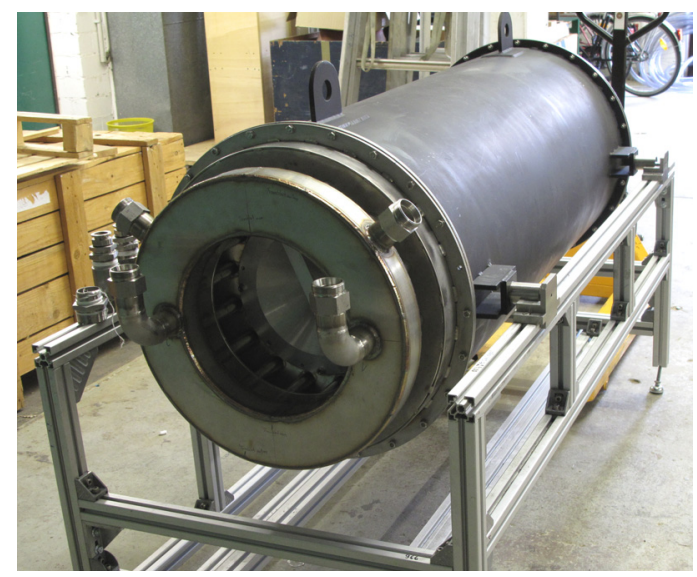

Fig.6 KIT-Prototype of an all stainless steel load

\section{Cryomagnet for frequency step tunable gyrotron}

Parallel to the development of a frequency step tunable gyrotron [6] the procurement of a superconducting magnet had been started at KIT, together with the corresponding power supplies. The aim is to achieve a 
quick frequency change at a rate of $6.6 \mathrm{GHz} / \mathrm{s}$, with a repetition-rate of 1 every 10 seconds.

The magnet is specified to be used up to 7.2 Tesla (corresponding to an electron cyclotron frequency of $174.2 \mathrm{GHz}$ ), for gyrotrons operating up to $180 \mathrm{GHz}$. Table 1 summarizes the main parameters for the magnet.

Table 1: Main parameters of fast tunable superconducting magnet.

\begin{tabular}{|l|l|l|}
\hline \multicolumn{2}{|l|}{ Max. Field } & 7.2 Tesla \\
\hline \multicolumn{2}{|l|}{ rate of Field change (up to 5.665 T) } & $\geq 0.264 \mathrm{~T} / \mathrm{s}$ \\
\hline $\begin{array}{l}\text { Warm Borehole } \\
\text { Diameter }\end{array}$ & everywhere & $\geq 220 \mathrm{~mm}$ \\
\cline { 2 - 3 } & top $135 \mathrm{~mm}$ & $\geq 275 \mathrm{~mm}$ \\
\hline $\begin{array}{l}\text { max. horizontal displacement of field } \\
\text { axis referred to geometrical axis }\end{array}$ & $\leq 0.1 \mathrm{~mm}$ \\
\hline $\begin{array}{l}\text { L-He volume } \\
\text { and boil off rate } \\
\text { requirements } \\
\text { without refill }\end{array}$ & operation at $6.86 \mathrm{~T}$ & $\geq 120 \mathrm{~h}$ \\
\cline { 2 - 3 } & hold time (0T) & $\geq 190 \mathrm{~h}$ \\
\hline
\end{tabular}

Figure 7 gives the coil arrangement chosen by the supplier of this magnet. The letters indicate to which group a coil belongs (see column 1 of table 2). The desired fast field change is obtained by acting on the AC mode coils (AC). The direction of the field in the emitter region $(\mathrm{z}=28 \mathrm{~mm})$ can be modified by acting on the Gun Taper coils $(\mathrm{G})$ and the Gun Trim Coils (T). The structure inside the $\mathrm{r}=0.1 \mathrm{~m}$ radius indicates the gyrotrons gun (up to the cavity).

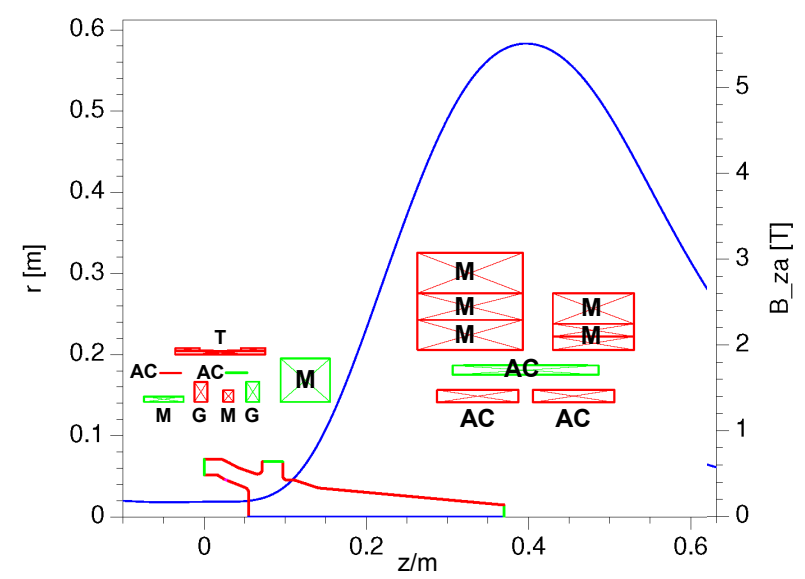

Fig.7 Coil configuration and Field-profile of the cryo-magnet for the fast frequency step tunable gyrotron

The magnet has demonstrated its maximum field capability of 7.2 Tesla and has sustained without problems the magnetic-field sweeps as specified. The helium boil-off rate was also within the specifications, which allow for an excellent stand-alone time in excess of 1 week for the magnet. Final acceptance however is delayed due to problems with the accuracy of the magnetic field alignment.

Table 2 gives the relevant power supply data for the 4 different coil systems. The power supply for the AC- coils is the most complex and powerful one; it operates in 4 quadrants and is capable of changing the current in the $\mathrm{AC}$ coil by $20 \mathrm{~A}$ in 0.5 seconds with a repetition rate of once every 10 seconds.

Table 2: Coil systems and required Power Supplies

\begin{tabular}{|l|l|l|l|}
\hline Power Supply & Inductance & Voltage & Current \\
\hline Main Coil (M) & $574 \mathrm{Hy}$ & $\pm 10 \mathrm{~V}$ & $+100 \mathrm{~A}$ \\
\hline AC-Coil (Mode) (AC) & $2.3 \mathrm{Hy}$ & $\pm 100 \mathrm{~V}$ & $\pm 125 \mathrm{~A}$ \\
\hline Gun Taper Coil (G) & $3.3 \mathrm{Hy}$ & $\pm 5 \mathrm{~V}$ & $\pm 20 \mathrm{~A}$ \\
\hline Gun Trim Coil (T) & $1.3 \mathrm{Hy}$ & $\pm 20 \mathrm{~V}$ & $\pm 10 \mathrm{~A}$ \\
\hline $\begin{array}{l}\text { Dipole Coils } \\
\text { (transverse field) }\end{array}$ & - & $5 \mathrm{~V}$ & $84 \mathrm{~A}$ \\
\hline
\end{tabular}

\section{Acknowledgments}

The coil arrangement shown in Fig. 4 has been devised by Cryomagnetics Inc., Oak Ridge, TN, USA. Thanks to our designer Mr. J. Weggen for drawings of the KIT load and to the remaining co-authors for providing input to this paper.

\section{References}

[1] G. Gantenbein et al., $22^{\text {nd }}$ Joint Russian-German Meeting on ECRH and Gyrotrons, June29-July 5, 2010

[2] A. Schlaich et al., .11th IEEE International Vacuum Electronics Conference IVEC2010 - in press

[3] T. Rzesnicki et al., $11^{\text {th }}$ IEEE International Vacuum Electronics Conference (IVEC 2010), May 18-20, 2010, Monterey, CA, USA - in press

[4] Andreas Schlaich: KIT Scientific Reports 7541, KIT Scientific Publishing 2010, ISSN 1869-9669

[5] S. Kern, $35^{\text {th }}$ international Conference on Infrared, Millimeter and Terahertz Waves, Sept. 5-10, 2010, Rome. Electronic Proceedings - in press

[6] M. Thumm, Fusion Engineering and Design, Vol. 53, Issues 1-4, January 2001, p.407-421 